\title{
Frequency of Celiac Disease in Children Presented with Liver Disease at a Tertiary Care Center
}

\author{
FAIKA HUSSAIN ${ }^{1}$, ASM BAZLUL KARIM ${ }^{2}$, MD RUKUNUZZAMAN ${ }^{3}$, SYEDAAFRIAANWAR $^{4}$, KANIZ $^{2}$ \\ SULTANA $^{5}$, NADIRA MOSABBIR ${ }^{6}$, MD. ARIFUL HAQUE ${ }^{7}$
}

\begin{abstract}
Background: Celiac disease is (CD) a genetically determined chronic inflammatory disease induced by an environmental precipitant. It is a multisystem disease and can develop at any point of time during life in genetically susceptible individuals upon ingestion of wheat gluten and related cereal proteins. The onset of symptoms in the atypical form generally occurs between 4-15 years of age. Diagnosis of CD with extraintestinal manifestations is frequently missed as it presents without diarrhoea.
\end{abstract}

Objective: To observe the frequency of celiac disease in children with liver disease.

Methods: This cross sectional study was conducted at BSMMU from January 2014 to June 2015. A total of 59 children (age 18 months to16 years) with clinical and biochemical features of liver disease were initially enrolled for the study. Their clinical history, examination findings and investigation reports were recorded in a data collection sheet and informed consent was obtained from parents. Routine investigations, liver function tests, tissue transglutaminase tTG (IgA), total IgA. were done. After exclusion of other causes of liver disease endoscopy of upper gastrointestinal tract (GIT) was done on patients who were $t T G$ (titer of $>50 \mathrm{iu} / \mathrm{mL}$ ) positive. Patients who were tTG negative but found IgA deficient (1 patient) was also selected for upper Gl endoscopy and biopsy fragments were taken from second part of duodenum (D2) sent for histopathology.

Results: Mean age of studied children was $8.33 \pm 3.64$ years. Out of 59 children with liver disease, $32.2 \%$ were $t T G$ positive of whom female were $8(13.6 \%)$ and male 11 (18.6\%). Mean age at diagnosis of all patients with tTG positive group was $8.24 \pm$ 2.78 (range 4-12) years. Among 19 sero-positive patients, short stature was found in $57.9 \%$ children. Mean $\mathrm{Hb}$ level in tTG positive group was $8.83 \pm 2.64 \mathrm{gm} / \mathrm{dl}$ and in $\mathrm{tTG}$ negative group $(10.27 \pm 1.74 \mathrm{gm} / \mathrm{dl})$. Sixteen (84.2\%) out of $19 \mathrm{tTG}$ positive patients had raised S. ALT. Out of 19 tTG positive children, endoscopy was done in 15 cases (endoscopy could not be done in 4 patients due to persistently raised PT) along with 1 patient who was IgA deficient. Endoscopic changes were mosaic and scalloping of $D 2$ mucosa in 1 and 2 cases respectively. Histological changes compatible with $C D$ were found in $5(31.3 \%)$ patients. Marsh 3a category was found in $2(12.5 \%)$ cases and $3 b$ in $3(18.8 \%)$ cases.

Conclusion: In the present study, 32.2\% liver disease cases were found tTG positive. Histological changes compatible with $C D$ were found in about one-third cases. Screening for celiac disease may be included in the diagnostic tests for evaluation of liver disease in children.

Key words: Celiac disease, tissue transglutaminase tTG (IgA), duodenal (D2) biopsy, liver disease.

1. Registrar, Department of Pediatrics, Shaheed Suhrawardy Medical College Hospital.

2. Chairman, Department of Pediatric Gastroenterology, BSMMU, Dhaka.

3. Associate Professor, Department of Pediatric Gastroenterology, BSMMU, Dhaka.

4. Consultant, Gastroliver Hospital, Dhaka.

5. Outdoor Medical Officer Eye, Dhaka Medical College Hospital, Dhaka.

6. Outdoor Medical Officer ENT, Dhaka Medical College Hospital, Dhaka.

7. Outdoor Medical Officer, Institute of Cancer Research Hospital, Mohakhali, Dhaka.

Correspondence: Dr. Faika Hussain, Registrar, Department of Pediatrics, Shaheed Suhrawardy Medical College Hospital, Dhaka.

E-mail: hussainfaika@gmail.com 


\section{Introduction}

Celiac disease is a frequent, lifelong, primarily small intestinal enteropathy which is induced in genetically susceptible individuals after ingestion of wheat gluten. It is a multisystem disease and can develop at any point of time during life in genetically susceptible individuals upon ingestion of wheat gluten and related cereal proteins. ${ }^{1,2}$ Global prevalence is approximately $1-5 \%$ of the general population. ${ }^{3}$ Celiac disease affecting patients is now well recognized in Northern India and prevalence is 1 in $96(1.04 \%) .{ }^{4} \mathrm{CD}$ individuals may present gastrointestinal symptoms, extraintestinal symptoms or no sign or symptoms. About $50 \%$ of CD patients present extraintestinal or atypical symptoms, such as anaemia, osteoporosis, dermatitis herpetiformis, neurological problems and dental enamel hypoplasia. ${ }^{5}$

Until the late 1970s, the suspicion of celiac disease was based mainly on clinical symptoms such as diarrhea, malabsorption and weight loss. Interestingly, a growing proportion of new cases of $C D$ are being diagnosed in patients with extra intestinal manifestations. CD may affect several organs including kidney, skin, heart, nervous, endocrine and reproductive systems. ${ }^{6}$ However, liver injury is one of the most frequent $(15-55 \%)$ extra intestinal manifestations of the disease. ${ }^{6}$

Based on the clinical features, serology and biopsy, celiac disease is classified as: ${ }^{7}$

1. Classical celiac disease (dominated by gastrointestinal malabsorption)

2. Atypical celiac disease (with prominent extra intestinal symptoms and a few or no gastrointestinal symptoms)

3. Silent celiac disease (asymptomatic, positive serology \& villous atrophy) and

4. Latent celiac disease (asymptomatic, positive serology but normal duodenal biopsy).

The occurrence of liver impairment in CD is well established and must be regarded as one of the various extra intestinal presentations of gluten-sensitive enteropathy. ${ }^{6}$ At least $20 \%$ to $30 \%$ of patients with extraintestinal manifestations present with hepatic injury. There are 2 main forms of liver damage in CD: cryptogenic and autoimmune-mediated. Cryptogenic liver damage is more frequent; it is typically asymptomatic and is characterized by mild hypertransaminasemia. It is not associated with autoantibodies other than CD autoantibodies. ${ }^{8}$ Serology has become increasingly relevant to $C D$ diagnosis. It is likely that serology could identify CD in its early stages, before the appearance of a severe intestinal damage. The best strategy for $\mathrm{CD}$ serological screening is the detection of Anti tissue transglutaminase antibody (Anti-tTG $\lg \mathrm{A}$ ) and Anti endomysial antibody. These antibodies are the most sensitive \& specific tests for the diagnosis of $C D$. The tTG ( IgA ) is currently the test of choice and is widely available worldwide. Sensitivity and specificity for tTG $(\lg A)$ are both $98 \%$ and PPV is $34 \% .{ }^{9}$ Serum tTG (IgA) level may not be elevated in patient with deficient $\lg A$, so serum IgA level must also be measured to avoid a false negative result. ${ }^{10,11}$

$C D$ has become a wide spread public health problem. ${ }^{12}$ Case finding for celiac disease in patients with liver disease is both cost-effective and beneficial in terms of quality of life. Early detection of this disease can prevent many of its unwanted complications. The current treatment for celiac disease is strict gluten free diet for life, although there is the possibility of alternate treatments. In the gluten-free diet wheat, barley and rye are to be avoided. The diagnosis should be definitive (i.e. proven by biopsy). In patients with celiac disease, mortality rate is higher than the general population. The important fact is that this mortality can be reduced to that of the general population after 1-5 years on a gluten free diet. ${ }^{10,11}$ So from this clinical point of view, early detection of celiac disease with serological testing or duodenal biopsy should be included in liver disease panel.

To the best of knowledge, there is a limited study in Bangladesh to find out the frequency of celiac disease in children with clinical and or biochemical features of liver disease. So from a clinical point of view, investigation of liver disease patients for celiac disease may be a valid approach.

\section{Materials and Methods}

Patients attending the Paediatric Gastroenterology and Nutrition department having clinical and biochemical features of liver disease were enrolled for the study. Patients clinical characteristics retrieved from medical interview, physical examinations and careful analysis of patients records. Patients who met the inclusion criteria and gave written consent to participate in the study were included in the study. Parents and the patients were informed about the nature of the study, its usefulness and its 
consequences. All data collection was performed by the researcher herself according to a self developed data collection sheet. Blood samples were collected from all patients in a standardized manner. Samples were analyzed for hematological parameters (including $\mathrm{Hb} \%$ and total leukocyte count), liver function tests (including serum bilirubin, ALT and serum albumin), serum TTG IgA and total IgA. In children, liver disease is defined as patients having any clinical (on examination- icteric, ascites or any stigmata of liver disease if present) and biochemical features of liver disease (any abnormality of LFT-raised S. bilirubin, S. ALT, PT or decreased S. albumin) were enrolled for the study. It might be acute or chronic liver disease. In patients with positive anti tTG $(\lg A)$ (titer of $>50$ $\mathrm{iu} / \mathrm{mL}$ ) were selected for upper $\mathrm{GI}$ endoscopy and biopsy from second part of duodenum. Patient who is IgA anti tTG negative but clinically susceptible, after excluding all other causes of liver dysfunction were selected for evaluation of serum IgA. Patient with serum IgA deficiency was also selected for upper $\mathrm{GI}$ endoscopy and biopsy from second part of duodenum. After 6-8 hours of fasting condition endoscopy of upper GIT were done using an Olympus forward viewing video endoscope under topical lignocaine anesthesia. During endoscopy, at least 2 biopsy fragments were taken from second part of duodenum and kept in a formalin filled test tube and were sent to the pathology department for histopathology. The endoscope \& biopsy forceps were carefully cleaned and disinfected by immersing the scope in $2 \%$ glutaraldehyde for 20 minutes and then rinsing it with clean water. After half an hour of endoscopy and biopsy, patients were allowed to take at first liquid then semisolid and normal diet orally within two to three hours.

The small intestinal biopsy is considered the gold standard for diagnosis of CD using the modified Marsh criteria. ${ }^{13}$ According to the modified Marsh classification: normal mucosa is classified Marsh 0 , intraepithelial lymphocytosis (>30 IEL/100 enterocytes-deodenum) as Marsh I, intraepithelial lymphocytosis (>30 IEL/100 enterocytes-deodenum) and increased crypt hyperplasia as Marsh II, and intraepithelial lymphocytosis, increased crypt hyperplasia and villous atrophy as Marsh III. Stage III was splitted into three sub stages ( $a, b$ and $c)$. Type $3 a, 3 b$, and $3 c$ destructive lesion are characterized by mild, marked, and complete villous atrophy respectively.
Statistical analysis was done using the Statistical Package for Social Science 17.0 (SPSS; Chicago, Illinois) for Windows XP. Mann Whitney $U$ test was done to assess the difference between two means of two groups. Test of association between male and female was carried out by chi-square test. Parents or legal guardian of patients were informed and explained about the nature of the study and written consent was taken from them. The patients had every right not to participate in the study. If any problem, damage to health or any adverse effects occurred due to the study procedure the researcher was responsible for that. Ethical clearance was taken from Institutional Review Board of BSMMU.

\section{Results}

Most (47.5\%) of the children were in between 5 - 10 year age group. Mean age at diagnosis of all patients with ITG positive group was $8.24 \pm 2.78$ (range $4-12$ ) years and in tTG negative group it was $8.37 \pm 4.01$ (range 1.5-15) years. Out of 19 seropositive cases, male children, $11(57.9 \%)$ and female children, 8 $(42.1 \%)$ were positive for $\mathrm{tTG}(\lg A)$. There is no significant difference between male and female. Among the studied children height for age $Z$ score $<-$ 3 were seen in $19(32.2 \%)$ cases and $\geq-3$ in $40(67.8 \%)$ cases. It is statistically significant.

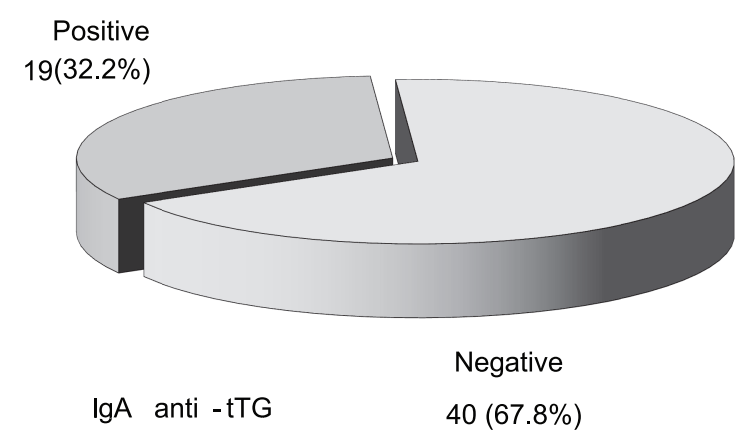

Fig 1: Results of tTG (IgA) ( $n=59)$

Table-I

Haemoglobin level in IgA anti tTG positive and negative children

\begin{tabular}{lccc}
\hline Haemoglobin & $\begin{array}{c}\text { tTG }(\operatorname{IgA}) \\
\text { positive } \\
(\mathrm{n}=19)\end{array}$ & $\begin{array}{c}\mathrm{tTG}(\operatorname{IgA}) \\
\text { negative } \\
(\mathrm{n}=40)\end{array}$ & $\begin{array}{c}\mathrm{p} \\
\text { value }\end{array}$ \\
\hline Mean $\pm \mathrm{SD}(\mathrm{gm} / \mathrm{dL})$ & $8.83 \pm 2.64$ & $10.27 \pm 1.74$ & $<0.05^{*}$ \\
Range & $1.40 \dagger 12.30$ & $6.30 \dagger 16.00$ & \\
\hline
\end{tabular}

Mann Whitney $U$ test was done to measure the level of significance. ${ }^{*}=$ Significant 
Among 19 seropositive patients, 16 (84.2\%) cases presented with raised S. ALT, 11 (57.9\%) with hypoalbuminaemia, 7 (36.8\%) with coagulopathy, 5 $(26.3 \%)$ with hyperbilirubinaemia and $4(21.0 \%)$ with jaundice.

After exclusion of other causes of liver disease endoscopy of upper gastrointestinal tract (GIT) was done on patients who were tTG (titer of $>50$ $\mathrm{iu} / \mathrm{mL}$ ) positive. Endoscopy was done in 15 cases (endoscopy could not be done in 4 patients due to persistently raised $\mathrm{PT}$ ) along with 1 patient who was IgA deficient. Endoscopic changes were mosaic and scalloping of D2 mucosa in 1 and 2 cases respectively and normal in others.

\section{Table-II}

Duodenal mucosal histological feature $(n=16)$

\begin{tabular}{|c|c|c|}
\hline $\begin{array}{l}\text { Histological } \\
\text { characteristics }\end{array}$ & Grade & No. (\%) \\
\hline IEL/100 enterocytes & $0(<30)$ & $5(31.3)$ \\
\hline \multirow[t]{2}{*}{ ofduodenum } & $1(>30)$ & $3(18.8)$ \\
\hline & $\begin{array}{l}\text { 2(>30 with crypt } \\
\text { hyperplasia) }\end{array}$ & $8(50)$ \\
\hline \multirow[t]{2}{*}{ Crypt hyperplasia } & Absent & $8(50)$ \\
\hline & Present & $8(50)$ \\
\hline \multirow[t]{5}{*}{ Villous atrophy } & $\begin{array}{l}3 a(>30 \text { IEL }+ \\
\text { crypthyperplasia }+ \\
\text { mild atrophy) }\end{array}$ & $2(12.5)$ \\
\hline & $3 b(>30+$ & $3(18.8)$ \\
\hline & $\begin{array}{l}\text { crypthyperplasia }+ \\
\text { moderate atrophy ) }\end{array}$ & \\
\hline & $3 c(>30+$ & 0 \\
\hline & $\begin{array}{l}\text { crypthyperplasia + } \\
\text { complete atrophy) }\end{array}$ & \\
\hline
\end{tabular}

Among 16 suspected CD patients, histological changes of intra epithelial lymphocytes (IEL) $>30 / 100$ enterocytes of duodenum were found in $11(68.8 \%)$ patients and normal in $5(31.3 \%)$ patients. Crypt hyperplasia were present in $8(50 \%)$ cases and absent in others. Marsh 3a category was found in $2(12.5 \%)$ cases and $3 b$ in $3(18.8 \%)$ cases.

\section{Discussion}

Celiac disease is (CD) a genetically determined chronic inflammatory disease induced by an environmental precipitant. The prevalence of hypertransaminasemia in patients with $C D$ ranges between $15 \%$ and $61 \%$, with the highest prevalence observed in children. Conversely, the prevalence of $C D$ in patients with unexplained hypertransaminasemia is $10 \%$, a finding that justifies screening for $C D$ in all patients with abnormal liver biochemical test results. ${ }^{8}$

The onset of symptom in the atypical form generally occurs between 4-15 years. This form commonly presents with short stature and refractory anaemia. None of the children had past history of chronic diarrhea. ${ }^{14}$

In this study, frequency of celiac disease was evaluated among children with liver disease attending the Paediatric Gastroenterology and Nutrition department, BSMMU.

In the present study, 19 (32.2\%) of 59 children had positive tTG $(\operatorname{lgA})$ and mean age of 59 children was $8.33 \pm 3.64$ years. In a study in India, mean age at diagnosis of CD was $10.8 \pm 2.2$ (range 4-15) years, ${ }^{14}$ and which is similar to the present study. Another study in India showed that CD with extraintestinal menifestations usually seen in older children or adolescent. ${ }^{15}$

Out of 19 patients with positive tTG (IgA), male were 11 and female 8 . Of 37 male patients, $29.7 \%$ and among 22 female children, $36.4 \%$ were positive for tTG (IgA). In the present study, girls were more likely than boys to had $C D$. There is no significant difference between male and female which is identical to the findings reported in Sahara ${ }^{16}$ where it has been shown that a higher proportion of females were found to be positive for $C D$ as compared to males. In the present study, it is shown that although the percentage of CD was more in female, sample size of male patients was greater in relation to female. This may be due to under reporting of symptoms in female patient in our country.

Among the studied children height for age $Z$ score <3 were seen in 19 (32.2\%) cases and $\geq-3$ in 40 (67.8\%) cases. It is statistically significant. Among 19 seropositive patients, 11 (57.9\%) presented with short stature which is similar to the study done in India. ${ }^{14}$ In that study, it has been shown that most of the patients of $C D$ with extraintestinal manifestation commonly presented with short stature (4 out of 7 patients).

Histological changes compatible with CD were found in $5(31.3 \%)$ patients. Marsh 3a category was found in $2(12.5 \%)$ cases and $3 b$ in $3(18.8 \%)$ cases. In a 
study that was done in 4 cities in China, it has been reported that 2 patients with positive serology had no mucosal lesions. ${ }^{17}$ One explanation regarding this finding may be that, a patient may have CD with normal duodenal biopsy and it may be due to patchy involvement of the duodenum. Another possibility is that a patient may have latent $C D$ together with some degrees of gluten sensitivity. Another study done in India, ${ }^{18}$ it has been reported that a good proportion of Indian children with $C D$ had mild to moderate histological changes (Marsh 1 and 2).

Early screening and identification of $C D$ in at-risk groups can increase the level of awareness and capacity of physicians in recognizing various clinical presentations of $C D$ and allow them to treat patients accordingly.

\section{Limitations of the study}

Study was conducted on small sample size and no control patients were taken in this study.

Other serology was not investigated such as antiendomysial antibody and antigliadin antibody.

\section{Conclusion}

It can be concluded that celiac disease is an important cause of liver disease in the studied population which often remains undiagnosed. So, screening of celiac disease with serological testing or duodenal biopsy should be included in the diagnostic tests for evaluation of liver disease in children.

\section{References}

1. Denham JM, Hill ID. Celiac Disease and Autoimmunity: Review and Controversies. Current Allergy Asthma Report. 2013; 13: 347-53.

2. Shamir R. Heyman MB, Koning F, Wijimenga C, Achury JG. Catassi C, et al. Celiac Disease: Past, Present, and Future Challenges Dedicated to the Memory of Our Friend and Colleague, Prof David Branski (1944-2013). Journal of Pediatric Gastroenterology and Nutrition. 2014; 59 (supplement 1): 17-20.

3. Rodrigo R. Celiac Disease. World Journal of Gastroenterology. 2006; 12: 6585-93.

4. Makharia GK, AhujaV, Verma AK. Prevalence of Celiac Disease in the Northern Part of India. Journal of Gastroenterology \& Hepatology. 2011; 26: 894-900.

5. Rampertab SD, Pooran N, Brar P, Singh P, Green $\mathrm{PH}$. Trends in the presentation of Celiac disease. American Journal of Medicine. 2006; 119: 355 e9-14.
6. Zali MR, Nejad MR, Rostami K, Alavian SM. Liver Complications in Celiac Disease. Hepatitis Monthly. 2011; 11: 333-41.

7. Gujral N, Freeman, HJ, Thomson ABR. Celiac disease: Prevalence, diagnosis, pathogenesis and treatment. World Journal of Gastroenterology. 2012; 18: 6036-59.

8. Mounajjed T. Oxentenko A. Shmidt E, Smyrk T. The Liver in Celiac Disease. American Journal of Clinical Pathology. 2011; 136: 128-37.

9. Leffler DA, Schuppan D. Update on serologic testing in celiac disease. American Journal of Gastroenterology. 2010; 105: 2520-24.

10. Green PH, Cellier C. Celiac Disease. New England Journal of Medicine. 2007; 357: 1731- 43.

11. Green PHR, Jabri B. Coeliac Disease. The Lancet. 2003; 362: 383-91.

12. Malekzadeh R, Sachdev A, Fahid AA. Celiac disease in developing countries: Middle East, India and North Africa. Best Practice and Research Clinical Gastroenterology. 2005; 19: 351-58.

13. Oberhuber G, Granditsch G, Vogelsang H. The histopathology of celiac disease: time for a standardized report scherme for pathologists. European Journal of Gastroenterology and Hepatology. 1999; 11: 1185-94.

14. Puri AS, Garg S, Monga R. Tyagi P, Saraswat MK. Spectrum of Atypical Celiac Disease in North Indian Children. Indian Pediatrics. 2004; 41: 822-27.

15. Sharma A, Poddar U, Yachha SK. Time to recognize atypical celiac disease in Indian children. Indian Journal of Gastroenterology. 2007; 26: 269-73.

16. Catassi C, Ratsch KM, Gandolfi L, Pratesi R, Fabinai E, El Asmar R, et al. Why is celiac disease endemic in the people of the Sahara? Lancet. 1999; 354: 647-48.

17. Wang $X Q$, Liu $W, X u C D$, Mei $H$, Gao Y, Peng $\mathrm{HM}$, et al. Celiac disease in children with diarrhea In 4 cities in China. Journal of Pediatric Gastroenterology and Nutrition. 2011; 53: 368-70.

18. Bhatnagor S, Gupta SD, Mathur SM. Celiac disease with mild to moderate histologic changes is a common case of chronic dirrhoea in Indian children. Journal of Pediatric Gastroenterology and Nutrition. 2005; 41: 20409. 